\title{
Is there an intermediate massive black hole in the Galactic center: Imprints on the stellar tidal-disruption rate
}

\author{
Xian Chen ${ }^{1}$, \& F. K. Liu ${ }^{2}$
}

\begin{abstract}
It has been suggested that an intermediate-massive black hole (IMBH) with mass $10^{3-5} M_{\odot}$ could fall into the galactic center (GC) and form a massive black hole binary (MBHB) with the central supermassive black hole, but current observations are not sensitive to constrain all mass and distance ranges. Motivated by the recent discovery that MBHBs could enhance the rate of tidal-disruption events (TDEs) of stellar objects, we investigate the prospect of using stellardisruption rate to probe IMBHs in the GC. We incorporated the perturbation by an IMBH into the loss-cone theory and calculated the stellar-disruption rates in the GC. We found that an IMBH heavier than $2000 M_{\odot}$ could distinguishably enhance the stellar-disruption rate. By comparing observations of Sgr A* with the fall-back model for stellar debris, we suggested that the TDE rate in our Galaxy should not significantly exceed $0.002 \mathrm{yr}^{-1}$, therefore a fraction of the parameter space for the IMBH, concentrating at the high-mass end, can already be excluded. To derive constraint in the remaining parameter space, it is crucial to observationally confirm or reject the stellar-disruption rate between $10^{-4}$ and $10^{-2} \mathrm{yr}^{-1}$, and we discussed possible strategies to make such measurements.
\end{abstract}

Subject headings: black hole physics - Galaxy: center - methods:analytical X-rays: bursts

\section{Introduction}

It has been suggested that in the center of our own Galaxy, besides an unambiguously detected supermassive black hole $(\mathrm{SMBH})$ of $4.3 \times 10^{6} M_{\odot}$ whose location coincides with Sgr $\mathrm{A}^{*}$ (Genzel et al. 2010, and references therein), there might be another intermediate massive

\footnotetext{
${ }^{1}$ Kavli Institute for Astronomy and Astrophysics, Peking University, 100871 Beijing, China; chenxian@pku.edu.cn

${ }^{2}$ Department of Astronomy, Peking University, 100871 Beijing, China; fkliu@pku.edu.cn
} 
black hole (IMBH), which is brought in by an infalling satellite galaxy (Lang et al. 2011) or core-collapse star cluster (Portegies Zwart et al. 2006). The existence of massive black hole binary $(\mathrm{MBHB})$ in the Galactic center ( $\mathrm{GC}$ ) would ease the tension between stellar dynamics and some observations, e.g. the formation of a young stellar disk inside 10" (about 4 pc), the central hole of size $5^{\prime \prime}$ discovered in the old stellar population, the apparent high velocity dispersion of a stellar association IRS 13E located at 3.5" from Sgr A*, and the random orientation of the S-stars inside 1" (see Genzel et al. 2010, for a review).

However, there has been no conclusive observational evidence for or against an IMBH at the galactic center. Based on the proximity of Sgr A* to the center of the Galactic nuclear cluster (Yu \& Tremaine 2003), and more exclusively on the proper motion of Sgr A* (Hansen \& Milosavljević 2003; Reid \& Brunthaler 2004), most parameter space can be excluded for IMBHs of masses $M_{\mathrm{IMBH}} \gtrsim 2 \times 10^{3} M_{\odot}$ residing at distances $d>2$ milliparsec $(\mathrm{mpc})$ from Sgr $\mathrm{A}^{*}$. Slight improvement on the constraint can be obtained by taking into account the orbital stability of S-stars, which mostly excludes IMBHs of masses $M_{\mathrm{IMBH}} \gtrsim 10^{5} M_{\odot}$ at $0.3 \mathrm{mpc} \lesssim d \lesssim 10 \mathrm{mpc}$ (Yu \& Tremaine 2003), and $2 \times$ $10^{3} M_{\odot} \lesssim M_{\mathrm{IMBH}} \lesssim 4 \times 10^{3} M_{\odot}$ at $2 \mathrm{mpc} \lesssim d \lesssim 10 \mathrm{mpc}$ (Gualandris \& Merrit 2009). Besides, the short coalescence timescale $\left(\lesssim 10^{7} \mathrm{yr}\right)$ due to gravitational wave radiation also argues against IMBHs of any mass within a distance of $d \sim 0.1 \mathrm{mpc}$ from Sgr A* (Hansen \& Milosavliević 2003). Nevertheless, not all parameter space is excluded (e.g. see Figure 13 in Gualandris \& Merritt 2009). The low-mass end at $M_{\mathrm{IMBH}}<2 \times 10^{3} M_{\odot}$ is currently unaccessible, except that the IMBH should have $d>0.1 \mathrm{mpc}$ to comply with the argument of coalescence timescale. Even in the high-mass end, it is not ruled out that an IMBH with $2 \times 10^{3} M_{\odot} \lesssim M_{\mathrm{IMBH}} \lesssim 4 \times 10^{4} M_{\odot}$ may reside at either $0.1 \mathrm{mpc} \lesssim d \lesssim 2 \mathrm{mpc}$ or $0.06\left(M_{\mathrm{IMBH}} / 10^{4} M_{\odot}\right)^{2} \mathrm{pc} \lesssim M_{\mathrm{IMBH}} \lesssim 3.2\left(M_{\mathrm{IMBH}} / 10^{4} M_{\odot}\right)$ pc.

Previous studies on the stellar orbits bound to MBHBs discovered that the three-body interactions could significantly boost the orbital eccentricities of the stars, partially due to the secular Lidov-Kozai effect (Ivanov et al. 2005) and more importantly due to chaotic interactions (Chen et al. 2009). Consequently, compared to that in the single-black-hole case, a larger fraction of stars could reach the so called "tidal radius" of the central SMBH to be tidally disrupted, resulting in a burst of stellar-disruption events (Chen et al. 2011; Wegg \& Nate Bode 2011). A stellar tidal-disruption event (TDE) will produce a powerful flare by releasing the gravitational energy of the stellar debris (Rees 1988; Ulmer 1999) and is observable in multiple electromagnetic bands ranging from radio to $\gamma$-ray (Komossa 2002; Gezari et al. 2009; van Velzen et al. 2011; Bloom et al. 2011; Burrows et al. 2011). Therefore, if IMBHs indeed reside in the GC, they should as well enhance the rate of TDEs, which may be imprinted in the emissions from the GC. 
In this paper we first study the stellar-disruption rate in the GC in the presence of an IMBH. Based on our results, we discuss the prospect of constraining the parameters of the hypothetical IMBH using the observational TDE rates. The outline of the paper is as follows. In Section 2 we develop the loss-cone theory to incorporate the perturbation by an IMBH, then in Section 3 we calculate the stellar-disruption rate in the GC including the effects of the IMBH. In Section 4, we investigate the observability of a TDE from GC based on the standard fall-back model for the stellar debris. Finally, we discuss our results in Section 5 .

\section{Loss-cone theory}

Around the SMBH in our own Galaxy, the stars with pericenter distances smaller than

$$
\begin{aligned}
r_{t} & \simeq r_{*}\left(\frac{M_{\bullet}}{m_{*}}\right)^{1 / 3} \\
& \simeq 10^{-5.4} \mathrm{pc}\left(\frac{M_{\bullet}}{10^{6.6} M_{\odot}}\right)^{1 / 3}\left(\frac{r_{*}}{R_{\odot}}\right)\left(\frac{M_{\odot}}{m_{*}}\right)^{1 / 3}
\end{aligned}
$$

are subject to tidal disruption (Hills 1975; Rees 1988), where $r_{*}$ and $m_{*}$ are, respectively, the radius and mass of star, and $R_{\odot}$ and $M_{\odot}$ refer to the solar values. For $m_{*}=M_{\odot}$ and $r_{*}=R_{\odot}$, this "tidal radius" is about 10 times greater than the Schwarzschild radius of the Galactic SMBH and has an angular size of 0.1 mas when viewed from the Earth. Tidal disruption creates a stellar-deficient region in the phase space of specific binding energy $\mathcal{E}$ and specific angular momentum J of stars. This region is conventionally referred to as the "loss cone" because of its cone-like geometry when the system is spherically symmetric (Frank \& Rees 1976; Lightman \& Shapiro 1977). In the following, we restrict our calculations to the stars bound to the SMBH and far from the tidal radius, because they dominate the tidal-disruption rate. This corresponds to an energy range of $\sigma^{2} \lesssim \mathcal{E} \ll G M_{\bullet} / r_{t}$, where $\sigma \simeq 75 \mathrm{~km} \mathrm{~s}^{-1}$ is the one-dimensional stellar velocity dispersion in the GC (Genzel et al. 2010). These stars are orbiting the SMBH on near-Keplarian orbits with semi-major axis $a=G M_{\bullet} / 2 \mathcal{E}$, and the greatest semi-major axis we consider is $a_{\max }=G M_{\bullet} / 2 \sigma^{2} \simeq 1.6 \mathrm{pc}$. Given $\mathcal{E}$, the maximum angular momentum is $J_{c}=G M_{\bullet} /(2 \mathcal{E})^{1 / 2}$ and the boundary of the loss cone by definition is at $J_{\mathrm{lc}} \simeq\left(2 G M_{\bullet} r_{t}\right)^{1 / 2}$.

The rate of tidal disruption is determined by the rate of stars diffusing into the loss cone, which is normally more efficient in the $J$ direction of the $\mathcal{E}-J$ phase space $(J=$ $|\mathbf{J}|)$. In the simplest case where the system has a single stellar population with mass $m_{*}$, suppose the successive mutual scattering between a star with energy $\mathcal{E}$ and the background stars on average induces an angular-momentum change $J_{D}(\mathcal{E})$ during one stellar orbital 
period $P(\mathcal{E})=2 \pi G M_{\bullet} /(2 \mathcal{E})^{3 / 2} \simeq 1.6 \times 10^{3}(a / 0.1 \mathrm{pc})^{3 / 2} \mathrm{yr}$, then the "two-body" relaxation timescale can be calculated with $T_{2 \mathrm{~b}}(\mathcal{E}) \sim P J_{c}^{2} / J_{D}^{2}$. The dependence of $T_{2 \mathrm{~b}}$ on the square of angular momentum reflects that the two-body scattering is a random process, so $J_{D}$ sums up incoherently. Given the number of stars $n(\mathcal{E}) d \mathcal{E}$ in the energy range $\mathcal{E} \sim \mathcal{E}+d \mathcal{E}$, the loss-cone filling rate is proportional to $n(\mathcal{E}) d \mathcal{E} / T_{2 \mathrm{~b}}(\mathcal{E})$. More careful analysis, taking into account the detailed distribution function $f(\mathcal{E}, J)$ at the loss-cone boundary and the fact that stars can be deflected into and out of the loss cone during one $P(\mathcal{E})$ when $J_{D} \gg J_{\text {lc }}$, gives the following form for the loss-cone filling rate due to two-body relaxation:

$$
\mathcal{F}_{2 \mathrm{~b}}(\mathcal{E}) d \mathcal{E}=j_{D}^{2} n(\mathcal{E}) d \mathcal{E} / P(\mathcal{E})
$$

where

$$
j_{D}^{2} \equiv \min \left[J_{\mathrm{lc}}^{2} / J_{c}^{2},\left(J_{D} / J_{c}\right)^{2} / \ln \left(J_{\mathrm{lc}} / J_{c}\right)\right]
$$

(Young 1977; Perets et al. 2007). To calculate $\left(J_{D} / J_{\mathrm{lc}}\right)^{2}$, we adopted Equation $(14 \mathrm{~d})$ in Bahcall \& Wolf (1977).

For stars within about 0.1 pc from the Galactic SMBH, another efficient relaxation process is resonant relaxation (RR), a coherent change of $J$ driven by the torque exerted by the grainy gravitational field of finite number of stars (Rauch \& Tremaine 1996; Rauch \& Ingalls 1998). Our scheme to calculate the loss-cone filling rate due to $\mathrm{RR}$ is analogous to that in Hopman \& Alexander (2006a), but we also revised their formula to include the dependence of RR on stellar orbital eccentricity $e=\left[1-\left(J / J_{c}\right)^{2}\right]^{1 / 2}$ (Gürkan \& Hopman 2007). In the standard case with a single black hole $(\mathrm{BH})$, the timescale for coherent variation of $J$ by $\mathrm{RR}$ is limited by the orbital precession timescales induced by general relativity (GR) and by the non-Keplerian potential of the surrounding stellar cusp. Given $P(\mathcal{E})$ and $\epsilon=\left(1-e^{2}\right)^{1 / 2}$ for a stellar orbit, we calculate $t_{\mathrm{GR}}=\left(2 \epsilon^{2} / 3\right)\left(a / r_{S}\right) P(\mathcal{E})$ for the GR precession timescale and $t_{M} \simeq \epsilon^{-1}\left(M_{\bullet} / m_{*}\right) P(\mathcal{E}) / N(>\mathcal{E})$ for the cusp-induced precession, where $r_{S}=2 G M_{\bullet} / c^{2}$ is the Schwarzschild radius and $N(>\mathcal{E})$ denotes the number of stars with energy greater than $\mathcal{E}$. Then the coherent variation timescale, $t_{\omega}$, can be derived from $1 / t_{\omega}=\left|1 / t_{\mathrm{GR}}-1 / t_{M}\right|$, the minus sign before $1 / t_{M}$ due to the opposite precession directions. During $t_{\omega}, J$ varies coherently by $\Delta J_{\omega} \simeq \dot{J}_{\mathrm{RR}} t_{\omega}$, where $\dot{J}_{\mathrm{RR}} \simeq 0.25 e N^{1 / 2}(>\mathcal{E}) G m_{*} / a$ is the RR torque exerted on the stellar orbit (Gürkan \& Hopman 2007). On longer timescale $t \gg t_{\omega}$, the coherence is broken due to orbital precession, therefore $J$ varies incoherently as $\Delta J \simeq \Delta J_{\omega}\left(t / t_{\omega}\right)^{1 / 2}$. The RR timescale to erase the initial angular momentum, $J=\epsilon J_{c}$, is then $T_{\mathrm{RR}} \sim\left(\epsilon J_{c} / \Delta J_{\omega}\right)^{2} t_{\omega}$, and more precisely

$$
T_{\mathrm{RR}}(\mathcal{E}, J) \simeq \frac{2.55 \epsilon^{2}}{e^{2}} \frac{P^{2}(\mathcal{E})}{N(>\mathcal{E})}\left(\frac{M_{\bullet}}{m_{*}}\right)^{2} \frac{1}{t_{\omega}}
$$


Gürkan \& Hopman 2007). The average relaxation timescale $\bar{T}_{\mathrm{RR}}$ for the stars with the same energy $\mathcal{E}$ is given by the integration of the equation $d J^{2} / J_{c}^{2}=d t / T_{\mathrm{RR}}(\mathcal{E}, J)$, i.e.,

$$
\bar{T}_{\mathrm{RR}}(\mathcal{E})=\int_{J_{\mathrm{lc}}^{2}}^{J_{c}^{2}} T_{\mathrm{RR}}(\mathcal{E}, J) d J^{2} / J_{c}^{2} .
$$

Then loss-cone filling rate due to $\mathrm{RR}$ can be calculated with

$$
\mathcal{F}_{\mathrm{RR}}(\mathcal{E}) d \mathcal{E}=n(\mathcal{E}) d \mathcal{E} / \bar{T}_{\mathrm{RR}}(\mathcal{E})
$$

If an IMBH with mass $M_{\mathrm{IMBH}}$ (the mass ratio of the IMBH-SMBH binary being $q \equiv$ $\left.M_{\mathrm{IMBH}} / M_{\bullet}\right)$ resides at a distance $d$ from $\operatorname{Sgr} \mathrm{A}^{*}$, the tidal force of the IMBH also exerts a torque on a stellar orbit, which induces an additional coherent variation of $J$ at the rate $\dot{J}_{K} \simeq J_{c} / T_{K}$, where

$$
T_{K}= \begin{cases}\frac{2}{3 \pi q}\left(\frac{a}{d}\right)^{-3} P(a) & (a \leq d / 2) \\ \frac{16 \sqrt{2}}{3 \pi q}\left(\frac{a}{d}\right)^{1 / 2} P(a) & (a>d / 2)\end{cases}
$$

is analogous to the Lidov-Kozai timescale (Lidov 1962; Kozai 1962) but also accounts for the stars in the chaotic regime with $a \sim d$ which dominates the loss-cone refilling (Chen et al. 2009, 2011). We note that during chaotic interactions the stellar orbits could reach extreme eccentricities $(e \simeq 1)$ irrespective of their initial inclinations or the $z$-components of angular momenta (Chen et al. 2011), which are fundamentally different from those interactions in the secular Lidov-Kozai mechanism and significantly increase the stellar reservoir for tidal disruption. Because of the extra nodal precession induced on the stellar orbit by the IMBH, the coherent variation of $J$ is limited by the new timescale $t_{\omega}^{\prime}=\left|1 / t_{K}+1 / t_{\mathrm{GR}}-1 / t_{M}\right|^{-1}$, where $t_{K} \simeq \epsilon T_{K}$ (Ivanov et al. 2005). During $t_{\omega}^{\prime}$, the variation of $J^{2}$, due to both $\mathrm{RR}$ and the IMBH perturber, is $\Delta\left(J_{\omega}^{\prime}\right)^{2}=\left(\dot{J}_{\mathrm{RR}}^{2}+\dot{J}_{K}^{2}\right)\left(t_{\omega}^{\prime}\right)^{2}$. The resulting timescale for erasing the initial $J$ is $T_{\text {co }}(\mathcal{E}, J) \simeq t_{\omega}^{\prime}\left(\epsilon J_{c}\right)^{2} / \Delta\left(J_{\omega}^{\prime}\right)^{2}$. Therefore, the coherent relaxation timescale due to both $\mathrm{RR}$ and an IMBH is

$$
\bar{T}_{\mathrm{co}}(\mathcal{E})=\int_{J_{\mathrm{lc}}^{2}}^{J_{c}^{2}} T_{\mathrm{co}}(\mathcal{E}, J) d J^{2} / J_{c}^{2},
$$

and the corresponding loss-cone filling rate is

$$
\mathcal{F}_{\mathrm{co}}(\mathcal{E}) d \mathcal{E}=\left(1-f_{\mathrm{ej}}\right) n(\mathcal{E}) d \mathcal{E} / \bar{T}_{\mathrm{co}}(\mathcal{E})
$$

We assume $f_{\text {ej }} \simeq 0.5$ to correct, to the zeroth order, the star loss due to slingshot ejection, since $f_{\mathrm{ej}}$ is a complex function of $q, a / d$, and the orbital eccentricity of the IMBH (Chen et al. 2011). 


\section{Stellar-disruption rate in the $\mathrm{GC}$}

To give $n(\mathcal{E}) d \mathcal{E}$ and $N(>\mathcal{E})$, we adopted a stellar distribution for the GC from Schödel et al. (2007), whose mass volume density has an outer Bachall-Wolf and an inner $\gamma=1.2$ power-law profile with a break radius at $r_{b} \simeq 0.22 \mathrm{pc}$. The corresponding stellar distribution function $f(\mathcal{E})$ scales as $f(\mathcal{E}) \propto \mathcal{E}^{-p}$, where $p=1 / 4(-0.3)$ for $\mathcal{E} \ll G M_{\bullet} / r_{b}\left(\mathcal{E} \gg G M_{\bullet} / r_{b}\right)$. The normalization for $f(\mathcal{E})$ is derived from $\rho(r)=4 \pi m_{*} \int_{0}^{G M_{\bullet} / r} \sqrt{2\left(G M_{\bullet} / r-\mathcal{E}\right)} f(\mathcal{E}) d \mathcal{E}$, then by definition we derive $n(\mathcal{E})=4 \pi^{2} J_{c}^{2} f(\mathcal{E}) P(\mathcal{E})$ and $N(\mathcal{E})=\int_{\mathcal{E}}^{G M_{\bullet} / 2 r_{t}} n(\mathcal{E}) d \mathcal{E}$. Using these quantities, as well as Equations (10) and (3) , we calculated the stellar-disruption rates in the GC for a grid of hypothetical IMBHs of different $M_{\mathrm{IMBH}}$ and $d$.

The dashed line in Figure 1 shows the stellar-disruption rate contributed by two-body relaxation at different semi-major axis, assuming $m_{*}=1 M_{\odot}$. Since $J_{D} \propto m_{*}^{1 / 2}$ and $r_{*} \propto m_{*}^{0.8}$ for main-sequence stars when $m_{*} \lesssim 20 M_{\odot}$ (Kippenhahn \& Weigert 1990), the rate $\mathcal{F}_{2 \mathrm{~b}}$ scales only mildly with $m_{*}$, as $1 / \ln m_{*}$ in the limit $J_{D} \ll J_{\text {lc }}$ and $m_{*}^{0.53}$ in the limit $J_{D} \gg J_{\text {lc }}$ (see Equation (4)). We do not show the contribution from the stars at $a>1.6 \mathrm{pc}$, because the Keplerian assumption would break down for these stars, but their contribution to the integrated loss-cone filling rate $\dot{N}=\int \mathcal{F}(\mathcal{E}) d \mathcal{E}$ is insignificant anyway. Inside the central $0.004 \mathrm{pc}$, the stellar mass is dominated by compact objects such as white dwarfs and stellarmass black holes (Hopman \& Alexander 2006b), therefore the rates inside 0.004 pc are not shown either. The total stellar-disruption rate due to incoherent relaxation, integrated over the range $0.004 \mathrm{pc}<a<1.6 \mathrm{pc}$ is $\dot{N}_{2 \mathrm{~b}} \simeq 3.5 \times 10^{-5} \mathrm{yr}^{-1}$, agrees well with the previous more sophisticated calculations (e.g. Merritt 2010).

The dotted line in Figure 1 shows the contribution to stellar-disruption rate by RR only, without the perturbation of an IMBH. Compared to two-body relaxation (dashed line), RR becomes more important at $a<0.1$ pc (also see Hopman \& Alexander 2006a), and the sharp decline of $\mathcal{F}(\mathcal{E}) \mathcal{E}$ inside $a \simeq 0.01 \mathrm{pc}$ is caused by the quenching of $\mathrm{RR}$ by relativistic precession. The integrated stellar-disruption rate is $\dot{N}_{\mathrm{RR}} \simeq 3.5 \times 10^{-6} \mathrm{yr}^{-1}$, and it does not depend on $m_{*}$ because both $n(\mathcal{E}) d \mathcal{E}$ and $\bar{T}_{\mathrm{RR}}(\mathcal{E})$ scales as $m_{*}^{-1}$.

If an IMBH with $M_{\mathrm{IMBH}}=10^{4} M_{\odot}$ resides in the $\mathrm{GC}$, the total stellar-disruption rates contributed by the coherent relaxations, i.e. RR plus IMBH perturbation, are shown in Figure 1 as the solid and dot-dashed lines. The rates are derived under the assumption $m_{*}=M_{\odot}$ and scale as $m_{*}^{-1}$ if $m_{*}$ varies. Comparing the solid lines with the dotted one, we found that the presence of the IMBH dramatically enhances the loss-cone filling rate. The integrated stellar-disruption rate derived form the solid lines, $\dot{N}_{\text {co }}$, increases with decreasing $d$ when $d \gtrsim 0.1$ pc. It reaches a maximum of $\dot{N}_{\text {co }} \simeq 1.1 \times 10^{-3} \mathrm{yr}^{-1}$ when $d \simeq 0.07 \mathrm{pc}$, which is a factor of 300 greater than the unperturbed value of $\dot{N}_{\mathrm{RR}}$, and 30 times higher than $\dot{N}_{2 \mathrm{~b}}$. 


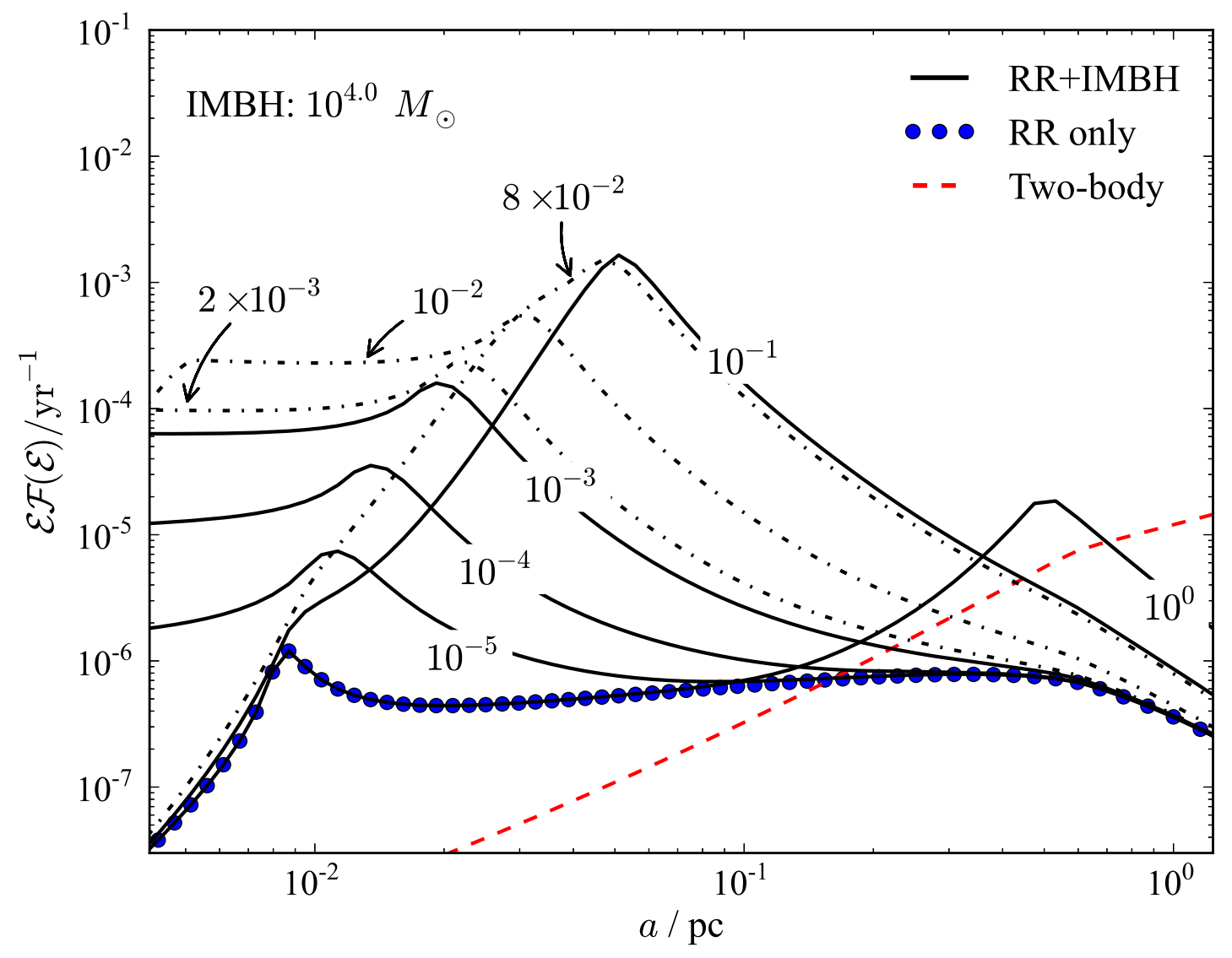

Fig. 1.- Contribution to loss-cone filling rate by stars at different semi-major axis, assuming $m_{*}=M_{\odot}$. Different stellar relaxation mechanisms are indicated with different line styles, i.e., dashed for two-body relaxation, dotted for $\mathrm{RR}$ only, and solid for RR+IMBH with $M_{\mathrm{IMBH}}=10^{4} M_{\odot}$. The annotations on the lines are the assumed distances $(d)$ in unit of pc for the IMBH. An IMBH with $10^{4} M_{\odot}$ at $d=\left(2 \times 10^{-3}, 10^{-2}, 8 \times 10^{-2}\right)$ pc is excluded by observations and thus the loss-cone filling rates due to the mechanisms $\mathrm{RR}+\mathrm{IMBH}$ are shown in dot-dashed lines. 
As $d$ further decreases from $d=0.07 \mathrm{pc}$, the integrated rate $\dot{N}_{\text {co }}$ becomes smaller, because the coherent variation of $J$ at $a \sim d$ becomes more susceptible to quench by relativistic precession.

Figure 2 (intensity map and contours) shows the total mass disruption rate, $\dot{M}=$ $m_{*}\left(\dot{N}_{2 \mathrm{~b}}+\dot{N}_{\mathrm{co}}\right)$, which is insensitive to the assumption of $m_{*}$, as a function of $M_{\text {IMBH }}$ and $d$. The dashed line indicates the parameter space that is excluded by the observed dynamics of S-stars and Sgr A* (Gualandris \& Merritt 2009). In general, $\dot{M}$ is a increasing function of $M_{\text {IMBH }}$ (because $\dot{N}_{\text {co }} \propto M_{\text {IMBH }}$ ), and given $M_{\text {IMBH }}$, the rate peaks at $d \sim 0.01-0.5$ pc. For example, when $M_{\mathrm{IMBH}}=\left(10^{3}, 10^{4}, 10^{5}\right) M_{\odot}$, the maxima of $\dot{M}$ are $(8.3,110,1600) \times$ $10^{-5} M_{\odot} \mathrm{yr}^{-1}$, occurring at $d=(0.03,0.07,0.3) \mathrm{pc}$. To the left-hand-side of the dashed line, where the possibility of an IMBH cannot be excluded by the current observations, the perturbed stellar-disruption rate ranges from $3 \times 10^{-5} M_{\odot} \mathrm{yr}^{-1}$ when $M_{\text {IMBH }} \leq 10^{3} M_{\odot}$ to as high as $4 \times 10^{-3} M_{\odot} \mathrm{yr}^{-1}$ when $M_{\mathrm{IMBH}} \simeq 10^{5} M_{\odot}$. We also derived $\dot{N}_{\text {co }}$ for $q=1 / 81$, $1 / 243$, and $1 / 729$ from the data of our previous scattering experiments in pseudo-Newtonian gravitational potentials (Chen et al. 2011). The resulting numerical rates agree with those from the above analytical calculations within a factor of 2 , despite many simplifications in our analytical model.

\section{Observability of TDEs in the GC}

The black contour in Figure $2\left(\dot{M} \simeq 10^{-4} M_{\odot} \mathrm{yr}^{-1}\right)$ indicates that an IMBH with $M_{\mathrm{IMBH}}>2000 M_{\odot}$, if currently resides in the $\mathrm{GC}$, would significantly raise the possibility of TDEs in the past $10^{2}-10^{4}$ years. The standard model for TDE (Rees 1988; Ulmer 1999) predicts that a fraction of the stellar debris will "fall back" to the periastron, forming an accretion disk and producing a shock-heated hot spot, whose initial luminosity should be close to or even exceeding the Eddington luminosity of the central $\operatorname{SMBH}\left(L_{E} \simeq 5.5 \times\right.$ $10^{44} \mathrm{erg} \mathrm{s}^{-1}$ for $\mathrm{GC}$ ). Is the hypothesized high stellar-disruption rate consistent with the observed quiescent state of Sgr A*?

In the framework of the "fall back model", the most bound seller debris has a binding energy of $\Delta \mathcal{E} \simeq k G M_{\bullet} r_{*} /\left(r_{t} / \beta\right)^{2}$ and will return to the periastron after a time delay of

$$
\begin{aligned}
t_{\min } & =2 \pi G M_{\bullet}(2 \Delta \mathcal{E})^{-3 / 2} \\
& \simeq 0.22 k^{-3 / 2} \beta^{-3}\left(m_{*} / M_{\odot}\right)^{-1}\left(r_{*} / R_{\odot}\right)^{3 / 2} \mathrm{yr}
\end{aligned}
$$

(Li et al. 2002), where $k \simeq 1--3$ accounts for the stellar spin at periastron before tidal disruption and $\beta \equiv r_{t} / r_{p} \sim 1$ denotes the ratio between the tidal radius $r_{t}$ and the pericenter distance $r_{p}$. About a fraction $f \sim 0.5$ of the stellar debris remains bound to the central SMBH 


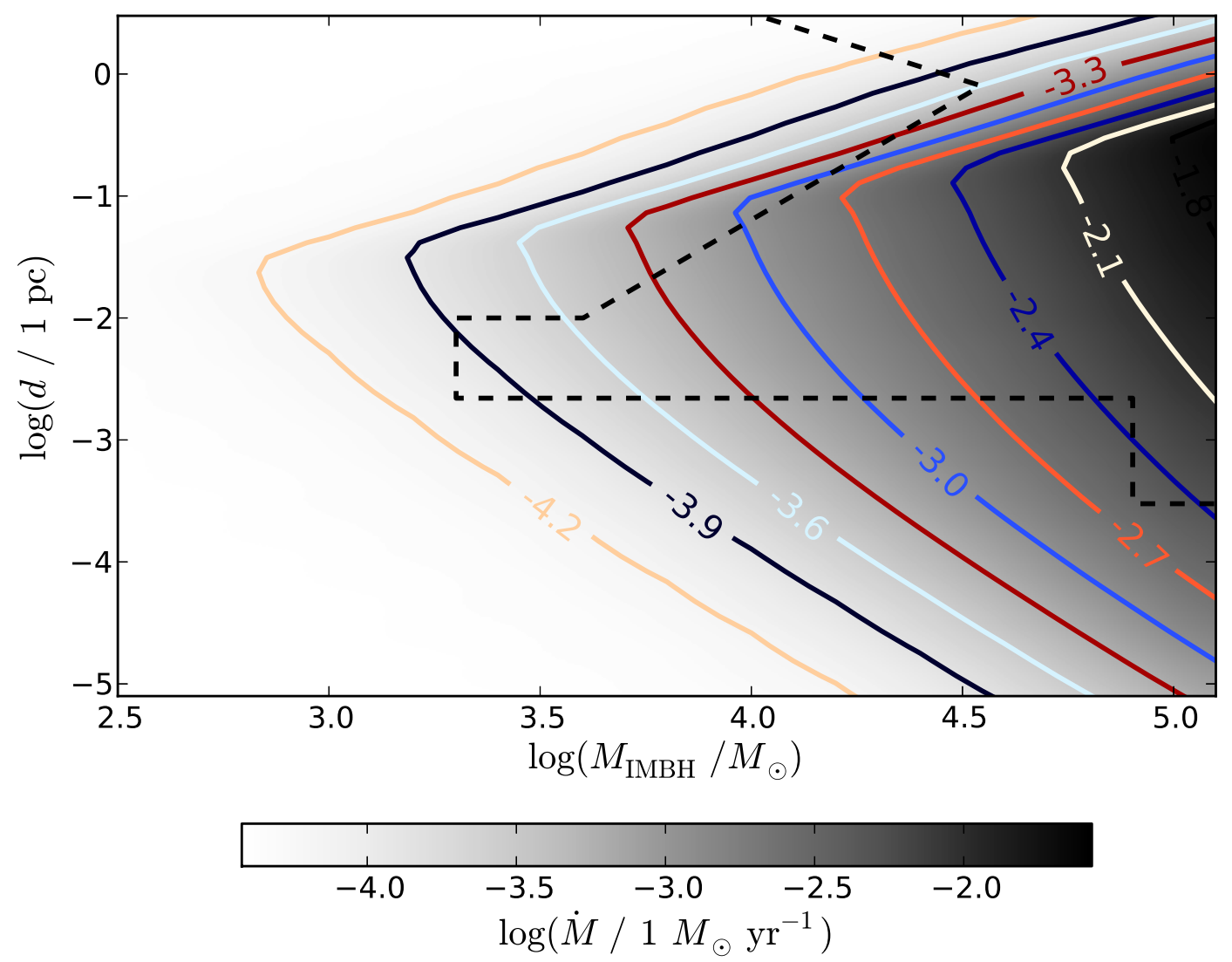

Fig. 2.- Integrated mass disruption rates (intensity map and contours), including contributions from both coherent and incoherent relaxations, for a grid of $M_{\mathrm{IMBH}}$ and $d$. The parameter space for IMBH excluded by the dynamics of S-stars and Sgr A* (Gualandris \& Merritt 2009) is the region to the right hand side of the dashed line. 
(Evans \& Kochanek 1989), and the resulting fall-back rate as a function of time is

$$
\begin{aligned}
\dot{M}_{\mathrm{fb}} & =\frac{2 f m_{*}}{3 t_{\min }}\left(\frac{t-t_{D}}{t_{\min }}\right)^{-5 / 3} \simeq 0.12 M_{\odot} \mathrm{yr}^{-1} k^{-1} \beta^{-2} \\
& \times\left(\frac{f}{0.5}\right)\left(\frac{m_{*}}{M_{\odot}}\right)^{1 / 3}\left(\frac{r_{*}}{R_{\odot}}\right)\left(\frac{t-t_{D}}{1 \mathrm{yr}}\right)^{-5 / 3}
\end{aligned}
$$

where $t_{D}$ denotes the time of stellar disruption and $t-t_{D} \geq t_{\min }$. We note that the fallback rate when $M_{\text {IMBH }}>4 \times 10^{4} M_{\odot}$ and $d>0.003$ pc is not calculated self-consistently, because in this case, according to Liu et al. (2009), $\dot{M}_{\mathrm{fb}}$ would be interrupted at $t-t_{D}>$ 10 yr. Anyway, this parameter space is excluded by the dynamics of Sgr A* and S-stars (Gualandris \& Merritt 2009). Because of mutual collisions when returning to the periastron, the fall-back material circularizes at a radius of about $2 r_{p}$, and the prompt release of the kinetic energy gives rise to a hot spot with bolometric luminosity

$$
\begin{aligned}
L_{\mathrm{hs}} & =\varepsilon \dot{M}_{\mathrm{fb}} c^{2} \simeq 9.5 \times 10^{42} \mathrm{erg} \mathrm{s}^{-1} \\
& \times k^{-1} \beta^{-1}\left(\frac{f}{0.5}\right)\left(\frac{m_{*}}{M_{\odot}}\right)^{2 / 3}\left(\frac{t-t_{D}}{1 \mathrm{yr}}\right)^{-5 / 3},
\end{aligned}
$$

where $\varepsilon \simeq G M_{\bullet} /\left(4 r_{p} c^{2}\right)$ is the conversion efficiency of kinetic energy into radiation and $c$ is the speed of light. The spectral energy distribution (SED) of the hot spot is mostly likely black-body (BB) because of the large opacity of the fall-back material. The effective temperature of the $\mathrm{BB}$ depends on the emission area, $A_{\mathrm{BB}}$, which is uncertain due to the current poor understanding of the structures of the colliding streams (Kochanek 1994; Kim et al. 1999). Since the vertical scale hight orthogonal to the orbital plane of the stellar stream is greater than $r_{*}$, the cross section for stream collision should not be much smaller than $2 \pi r_{*} r_{p}$, and the geometry factor $\zeta=A_{\mathrm{BB}} /\left(4 \pi r_{p}^{2}\right)$ for the emission area is likely greater than $r_{*} / r_{p} \sim 0.01$. For a typical value of $\zeta=0.1$, the effective temperature of the hot spot is

$$
\begin{aligned}
T_{\mathrm{BB}} & =\left(\frac{L_{\mathrm{bol}}}{\sigma_{S} A_{\mathrm{BB}}}\right)^{1 / 4} \simeq\left(\frac{\beta^{2} L_{\mathrm{bol}}}{4 \pi \sigma_{S} \zeta r_{t}^{2}}\right)^{1 / 4} \\
& \simeq 1.8 \times 10^{5} \mathrm{~K}\left(\frac{\beta}{k}\right)^{1 / 4}\left(\frac{f}{0.5}\right)^{1 / 4}\left(\frac{m_{*}}{M_{\odot}}\right)^{1 / 3} \\
& \times\left(\frac{\zeta}{0.1}\right)^{-1 / 4}\left(\frac{r_{*}}{R_{\odot}}\right)^{-1 / 2}\left(\frac{t-t_{D}}{1 \mathrm{yr}}\right)^{-5 / 12}
\end{aligned}
$$

where $\sigma_{S}$ is the Stefan-Boltzmann constant.

To get a sense of the brightness of the hot spot in the GC, we calculated $\dot{M}_{\mathrm{fb}}$ and $L_{\mathrm{hs}}$ as functions of $t-t_{D}$ with the fiducial parameters $\left(f, k, \beta, \zeta, r_{*}, m_{*}\right)=\left(0.5,1,1,0.1, R_{\odot}, M_{\odot}\right)$. 
We also increased $k$ and $\beta$ to $k=3$ and $\beta=5$ to estimate the lower limits to $\dot{M}_{\mathrm{fb}}$ and $L_{\mathrm{hs}}$. The results are given in Table 1 with the lower limits bracketed by parentheses. We found that 10 years after a TDE in the GC, the fall-back rate has already dropped to about 30-2600 times smaller than the Eddington rate, defined as $\dot{M}_{E} \equiv L_{E} /\left(0.1 c^{2}\right) \simeq 8.4 \times$ $10^{-2} M_{\odot} \mathrm{yr}^{-1}$. By this time, the bolometric luminosity of the hot spot is $4-5$ orders of magnitude smaller than the Eddington luminosity, when taking into account that $\varepsilon$ is much smaller than the typical value of 0.1 derived from the disk accretion theory. We note that when $t-t_{D} \gtrsim 100 \mathrm{yr}$, the bolometric luminosity contributed by the accretion disk produced by the circularized material is negligible because the fall-back rate is too low to support a radiatively efficient accretion disk (Cannizzo et al. 1990; Menou \& Quataert 2001). Even in the extreme case that the accretion flow can instantly drain the fall-back material (Ulmer 1999), the luminosity of the radiatively inefficient accretion flow is still as low as $10^{38} \mathrm{erg} \mathrm{s}^{-1}\left(\dot{M}_{\mathrm{fb}} / 10^{-4} M_{\odot} \mathrm{yr}^{-1}\right)^{2}$ (Naravan \& Yi 1995; Naravan et al. 1998). Observations of Sgr A* revealed that the bolometric luminosity is of order $10^{36-37} \mathrm{erg} \mathrm{s}^{-1}$ (Genzel et al. 2010), and the SED can be well modeled with a radiatively inefficient disk with an accretion rate of $10^{-6} M_{\odot} \mathrm{yr}^{-1}$ (Narayan et al. 1998). Detailed modeling of the accretion flow also indicates that the accretion rate inside 20 gravitational radii (smaller than the typical tidal radius) of the central SMBH is even smaller, ranging from $10^{-9}$ to $10^{-7} M_{\odot} \mathrm{yr}^{-1}$ (Shcherbakov et al. 2012, and references therein). These facts, when compared to $\dot{M}_{\mathrm{fb}}$ and $L_{\mathrm{hs}}$ derived from the fall-back model, suggest that TDEs are unlikely to have occurred in our own Galaxy within the last $4-5$ centuries.

We also calculated $T_{\mathrm{BB}}$ of the hot spot according to Equation (15). The results for the fiducial parameters (data without parentheses) and for $(k, \beta, \zeta)=(3,5,0.01)$ are also shown in Table 1. According to Wein's displacement law, the SED of the hot spot initially peaks at the UV band and will shift toward IR as $t-t_{D}$ increases to $10^{4}$ years. Since IR bands have relatively low extinctions toward the GC and are commonly used by the ground-based telescopes to monitor the GC, we calculated the monochromatic flux of the hot spot at a wavelength of $2.2 \mu \mathrm{m}$ ( $K$-band) with an extinction of $A=3$. The results are given in the last two rows of Table 1, where the data in parentheses correspond to non-fiducial parameters with $(k, \beta, \zeta)=(3,5,0.01)$. For other non-fiducial combinations of $\left(k, \beta, \zeta, f, m_{*}, r_{*}\right)$, one can use the scaling $F_{\nu} \propto L_{\text {bol }} / T_{\text {eff }}^{3}$ to derive the IR flux. The $K$ band flux from the radiatively inefficient accretion flow is very difficult to derive because of many unknown model parameters, but is typically smaller than $10\left(\dot{M}_{\mathrm{fb}} / 10^{-4} M_{\odot} \mathrm{yr}^{-1}\right)^{2} \mathrm{mJy}$ (Narayan et al. 1998). Observationally, the $K$-band flux of Sgr A* is measured to be $\lesssim 5$ mJy with occasional flares as bright as $30 \mathrm{mJy}$ (Morris et al. 2012, and references therein). Despite the uncertainties in the model predictions, we found the null hypothesis that a TDE occurred in the GC within the last $7-8$ centuries to be in severe conflict with the observed 
$K$-band flux of Sgr A*.

\section{Discussion}

In order to test the possibility and constrain the parameters of a hypothetical IMBH in the GC, we studied the effects of an IMBH on the stellar-disruption rate in the framework of the loss-cone theory. We found that an IMBH with mass greater than $2000 M_{\odot}$ could significantly enhance the stellar disruption rate in GC (Figure 1) because it coherently perturbs the angular momenta of stars in a fashion analogous to but more chaotic than the Lidov-Kozai effect. The maximum mass disruption rate increases with black hole mass as $10^{-3} M_{4} M_{\odot} \mathrm{yr}^{-1}$, where $M_{4}=M_{\mathrm{IMBH}} / 10^{4} M_{\odot}$, and is reached when the IMBH arrives at about $0.1^{\prime \prime} \sim 1^{\prime \prime}$ from Sgr $A^{*}$ (Figure 2). These results have already brought up some intriguing implications. For example, it is speculated that the high velocity dispersion of IRS 13E, a young stellar association locating at $0.14 \mathrm{pc}$ from $\mathrm{Sgr} \mathrm{A}^{*}$, may be induced by an embedding IMBH with mass greater than $10^{4} M_{\odot}$ (Maillard et al. 2004; Schödel et al. 2005; Fritz et al. 2010). If so, the mass disruption rate in the GC according to our calculation should be greater than about $5 \times 10^{-4} M_{\odot} \mathrm{yr}^{-1}$, up to about $0.02 M_{\odot} \mathrm{yr}^{-1}$ in the extreme case with $M_{\mathrm{IMBH}}=10^{5} M_{\odot}$. The results also imply that an IMBH in the GC would greatly increase the chance for us to detect infalling objects on the course toward Sgr A* (Gillessen et al. 2012).

Is the hypothesized high stellar-disruption rate consistent with the current quiescent state of Sgr A*? To estimate the set-off time for the most recent TDE in the GC, we calculated the evolution of the bolometric luminosity and the IR flux of a TDE in the GC using the fall-back model (Section 4 ). When comparing the model predictions with the observations of Sgr $\mathrm{A}^{*}$, we found that the current quiescent state of Sgr $\mathrm{A}^{*}$ only allows a stellar-disruption rate lower than about $0.002 \mathrm{yr}^{-1}$. According to this limit, the IMBH is unlikely to reside in the right-hand side of the $\log (\dot{M})=-2.7$ contour in Figure 2 , Interestingly, most part of this disfavored parameter space coincides with the space already excluded by the dynamics of Sgr A* and the S-stars (dashed line in Figure 2), confirming the previous constraints on the IMBH. In addition, the limit on $\dot{M}$ excludes a patch of the parameter space at $d \lesssim 0.002 \mathrm{pc}$ and $\dot{M} \gtrsim 0.002 M_{\odot} \mathrm{yr}^{-1}$, which was not reached by the dynamics of Sgr $\mathrm{A}^{*}$ and the S-stars. Furthermore, to comply with the limit on $\dot{M}$, the hypothetical IMBH in IRS $13 \mathrm{E}$ should not exceed $2 \times 10^{4} M_{\odot}$.

The above success encourages us to investigate the prospects of using stellar-disruption rates to probe even smaller and closer (to Sgr $\mathrm{A}^{*}$ ) IMBHs. For the parameter space of IMBH that is still allowed by the dynamics of Sgr $\mathrm{A}^{*}$ and the S-stars (Figure 2), the maximum 
stellar-disruption rate is $\dot{M} \simeq 10^{-3} M_{\odot} \mathrm{yr}^{-1}$ when $M_{\mathrm{IMBH}}=10^{4} M_{\odot}$ and $\dot{M} \simeq 10^{-4} M_{\odot} \mathrm{yr}^{-1}$ when $M_{\mathrm{IMBH}}=10^{3} M_{\odot}$, and these maxima occur at $d \sim 0.04 \mathrm{pc}$ (about 1 arcsec). Besides, at a distance as close as $d<0.002$ pc (about 50 mas), where there is nearly no constraint on $M_{\text {IMBH }}$ by the previous observations, the maximum $\dot{M}$ varies from about $4 \times 10^{-3} M_{\odot} \mathrm{yr}^{-1}$ for $M_{\mathrm{IMBH}}=10^{5} M_{\odot}$ to about $10^{-4} M_{\odot} \mathrm{yr}^{-1}$ for $M_{\mathrm{IMBH}}=2000 M_{\odot}$. Combining these results and taking into account that the stellar-disruption rate for a single $\mathrm{SMBH}$ in the GC is $\dot{N}_{2 \mathrm{~b}} \simeq 3.5 \times 10^{-5} \mathrm{yr}^{-1}$, we suggest that a stellar-disruption rate between $10^{-4}$ and $10^{-2} \mathrm{yr}^{-1}$, if identified in our own Galaxy, would be a strong evidence for an IMBH more massive than $2000 M_{\odot}$ lying in the central parsec of the GC. On the other hand, if the stellar-disruption rate higher than $10^{-4} \mathrm{yr}^{-1}$ is rejected by future observations, a large parameter space, which is not covered by the current constraints on the hypothetical IMBH, could be excluded.

A stellar-disruption rate of $10^{-2} \mathrm{yr}^{-1}$ is already too low to be measured directly from decadal observational cycles, therefore measuring a rate down to $10^{-4} \mathrm{yr}^{-1}$ requires special strategies. It is observed that the central tens of parsecs of the GC are populated by molecular clouds (MCs; see Genzel et al. 2010, for a review). A TDE at Sgr A* could leave imprints on the MC system in the form of reflected hard X-rays or fluorescent iron lines decades or even centuries after the outburst (Yu et al. 2011). A systematic search for such "light echoes" within $30 \mathrm{pc}$ from Sgr $\mathrm{A}^{*}$ indeed revealed a major flare as luminous as $3 \times 10^{39} \mathrm{erg} \mathrm{s}^{-1}$ in X-ray starting to decay at about 150 years ago (Morris et al. 2012, and references therein). These observations give a upper limit of $0.004 \sim 0.006 \mathrm{yr}^{-1}$ to the TDE rate in the GC because according to Equation (14) it takes at least $180-280$ years (depending on $k$ and $\beta$ ) for the luminosity of hot spot to drop below $3 \times 10^{39} \mathrm{erg} \mathrm{s}^{-1}$, not to mention that accounting extra X-ray contribution from an accretion disk would extend the time elapse $t-t_{D}$. To test whether the flare recurs every 200 300 years, it is crucial to look for a second light echo in the MC system $30-100$ pc away from Sgr A*. On longer timescales ranging from thousands to millions of years, the imprints of TDEs could be detected in the forms of enhanced electronpositron annihilation lines, high-energy gamma rays, and cosmic rays (Cheng et al. 2006, 2007, 2011). If absorbed by the bio-circle, the records of enhanced high-energy emissions could be preserved on the earth surface for thousands of years (Mivake et al. 2012). In order to constrain $\dot{M}$ in the GC over an extended timescale of $10^{4-6}$ years, we appeal for more efforts in measuring and modeling of the emissions in these high-energy bands.

We thank Roman Shcherbakov and Shuo Li for many helpful discussions. This work is supported by the Chinese national 973 program (2007CB815405) and the National Natural Science Foundation of China (NSFC11073002). F.K.L. also thanks the support from the China Scholarship Council (2009601137), and X.C. acknowledges the support from China Postdoc Science Foundation (2011M500001). 


\section{REFERENCES}

Bahcall, J. N., \& Wolf, R. A. 1977, ApJ, 216, 883

Bloom, J. S., Giannios, D., Metzger, B. D., et al. 2011, Science, 333, 203

Burrows, D. N., Kennea, J. A., Ghisellini, G., et al. 2011, Nature, 476, 421

Cannizzo, J. K., Lee, H. M., \& Goodman, J. 1990, ApJ, 351, 38

Chen, X., Madau, P., Sesana, A., \& Liu, F. K. 2009, ApJ, 697, L149

Chen, X., Sesana, A., Madau, P., \& Liu, F. K. 2011, ApJ, 729, 13

Cheng, K. S., Chernyshov, D. O., \& Dogiel, V. A. 2006, ApJ, 645, 1138

Cheng, K. S., Chernyshov, D. O., \& Dogiel, V. A. 2007, A\&A, 473, 351

Cheng, K.-S., Chernyshov, D. O., Dogiel, V. A., Ko, C.-M., \& Ip, W.-H. 2011, ApJ, 731, L17

Evans, C. R., \& Kochanek, C. S. 1989, ApJ, 346, L13

Frank, J., \& Rees, M. J. 1976, MNRAS, 176, 633

Fritz, T.K., Gillessen, S., Dodds-Eden, K., et al. 2010, ApJ, 721, 395

Genzel, R., Eisenhauer, F., \& Gillessen, S. 2010, RvMP, 82, 3121

Gezari, S., Strubbe, L., Bloom, J. S., et al., 2009, astro10: The Astronomy and Astrophysics Decadal Survey, 2010, 88 (arXiv:0903.1107)

Gillessen, S., Genzel, R., Fritz, T. K., et al. 2012, Nature, 481, 51

Gualandris, A., \& Merritt, D., 2009, ApJ, 705, 361

Gürkan, M. A., \& Hopman, C. 2007, MNRAS, 379, 1083

Hansen, B. M. S., \& Milosavljević, M. 2003, ApJ, 593, L77

Hopman, C., \& Alexander, T., 2006a, ApJ, 645,1152

Hopman, C., \& Alexander, T., 2006b, ApJ, 645, 133

Hills, J. 1975, ApJ, 254, 295

Ivanov, P. B., Polnarev, A. G., \& Saha, P., 2005, MNRAS, 358, 1361 
Kim, S. S., Park, M.-G., \& Lee, H. M. 1999, ApJ, 519, 647

Kippenhahn, R., \& Weigert, A., 1990, Stellar Structure and Evolution, (Berlin:Springer)

Kochanek, C. S., 1994, ApJ, 422, 508

Komossa, S., 2002, Rev. Mod. Astron., 15, 27

Kozai, Y. 1962, AJ, 67, 591

Lang, M., Holley-Bockelmann, K., Bogdanovic, T., Amaro-Seoane, P., \& Sesana, A. 2011, arXiv:1107.2923

Li, L., Narayan, R., \& Menou, K, 2002, ApJ, 576, 753

Lidov, M. L. 1962, Planet. Space Sci., 9, 719

Lightman, A. P. \& Shapiro, S. L., 1977, ApJ, 211, 244

Liu, F. K., Li, S., \& Chen, X., 2009, ApJ, 706, 133

Maillard, J. P., Paumard, T., Stolovy, S. R., \& Rigaut, F. 2004, A\&A, 423, 155

Menou, K., \& Quataert, E. 2001, ApJ, 562, L137

Merritt, D., 2010, ApJ, 718, 739

Miyake, F., Nagaya, K., Masuda, K., \& Nakamura, T. 2012, Nature, 486, 240

Morris, M. R., Meyer, L., \& Ghez, A. M., 2012, RAA, 12, 995

Narayan, R., Mahadevan, R., Grindlay, J. E., Popham, R. G., \& Gammie, C. 1998, ApJ, 492, 554

Narayan, R., \& Yi, I. 1995, ApJ, 452, 710

Perets, H. B., Hopman, C., \& Alexander, T., 2007, ApJ, 656, 709

Portegies Zwart, S. F., Baumgardt, H., McMillan, S. L. W., et al. 2006, ApJ, 641, 319

Rauch, K. P., \& Ingalls, B., 1998, MNRAS, 299, 1231

Rauch, K. P., \& Tremaine, S. 1996, NewA, 1, 149

Rees, M. J. 1988, Nature, 333, 523

Reid, M. J., \& Brunthaler, A. 2004, ApJ, 616, 872 
Schödel, R., Eckart, A., Iserlohe, C., Genzel, R., \& Ott, T. 2005, ApJ, 625, 111

Schödel R. et al., 2007, A\&A, 469, 125

Shcherbakov, R. V., Penna, R. F., \& McKinney, J. C. 2012, ApJ, 755, 133

Ulmer, A., 1999, ApJ, 514, 180

van Velzen, S., Farrar, G. R., Gezari, S., et al. 2011, ApJ, 741, 73

Wegg, C., \& Nate Bode, J. 2011, ApJ, 738, L8

Young, P. J. 1977, ApJ, 215, 36

Yu, Q., \& Tremaine, S. 2003, ApJ, 599, 1129

Yu, Y.-W., Cheng, K. S., Chernyshov, D. O., \& Dogiel, V. A. 2011, MNRAS, 411, 2002 
Table 1. Predictions from the Fall-back Model

\begin{tabular}{lclll}
\hline \hline$t-t_{D}($ years $)$ & 10 & $10^{2}$ & $10^{3}$ & $10^{4}$ \\
\hline$\dot{M}_{\mathrm{fb}}\left(M_{\odot} \mathrm{yr}^{-1}\right)$ & $10^{-2.6}$ & $10^{-4.3}$ & $10^{-5.9}$ & $10^{-7.7}$ \\
& $\left(10^{-4.5}\right.$ & $10^{-6.1}$ & $10^{-7.8}$ & $\left.10^{-9.3}\right)$ \\
$L_{\mathrm{hs}}\left(\mathrm{erg} \mathrm{s}^{-1}\right)$ & $10^{41.3}$ & $10^{39.6}$ & $10^{38.0}$ & $10^{36.3}$ \\
& $\left(10^{40.1}\right.$ & $10^{38.5}$ & $10^{36.8}$ & $\left.10^{35.1}\right)$ \\
$T_{\mathrm{BB}}\left(10^{3} \mathrm{~K}\right)$ & 79 & 31 & 12 & 4.5 \\
& $(160$ & 61 & 23 & $8.9)$ \\
$F_{\nu}(K)(\mathrm{mJy})$ & 160 & 56 & 15 & 2.0 \\
& $(35$ & 12 & 4.1 & $0.96)$ \\
\hline
\end{tabular}

